\title{
THE SUB-COULOMB ${ }^{12} \mathrm{C}+{ }^{12} \mathrm{C}$ RESONANCES IN A MICROSCOPIC ${ }^{12} \mathrm{C}+{ }^{12} \mathrm{C}, \alpha+{ }^{20} \mathrm{Ne},{ }^{8} \mathrm{Be}+{ }^{16} \mathrm{O}$ CLUSTER BASIS
}

\author{
Y. SUZUKJ * and K. T. HECHT \\ Physics Department, University of Michigan, Ann Arbor, Michigan 48109, USA ${ }^{\dagger}$
}

Received 23 March 1982

(Revised 1 June 1982)

\begin{abstract}
The low- $J$ resonances in the Coulomb barrier region of the ${ }^{12} \mathrm{C}+{ }^{12} \mathrm{C}$ system are investigated in the framework of a microscopic cluster model basis including ${ }^{12} \mathrm{C}+{ }^{12} \mathrm{C}, x+{ }^{20} \mathrm{Ne}$, and ${ }^{8} \mathrm{Be}+{ }^{16} \mathrm{O}$ fragment decompositions. Calculations are carried out in an orthogonality condition model approximation in which Pauli-forbidden components are properly excluded from the basis but in which the interaction among cluster fragments is approximated by a local potential, obtained from a gaussian NN interaction by a folding procedure leading to both spherical and $Q \cdot Q$ terms. Only minor adjustments of overall strength and fall-off parameters are introduced to gain a consistent picture of the low-energy spectrum in the separate rearrangement channels. The basis includes cluster relative motion excitations with oscillator quanta from 12 to 20 and is not quite rich enough to give a detailed quantitative comparison between theory and experiment. Predicted excitation energies are too high by $\sim 3 \mathrm{MeV}$ and predicted ${ }^{12} \mathrm{C}$ partial widths are too small to indicate a well-developed surface-peaked molecular character; but it does appear possible to identify a $5 \mathrm{MeV}$ region as the potential seat of the $0^{+}, 2^{+}, 4^{+}$resonances. The number and approximate spacing of the resonance fine structure components are in agreement with experiment.
\end{abstract}

\section{Introduction}

Since the first discovery ${ }^{1}$ ) of the narrow resonances near the Coulomb barrier in the ${ }^{12} \mathrm{C}+{ }^{12} \mathrm{C}$ system a large number of investigations, both experimental and theoretical, have been undertaken in an attempt to elucidate the nature of these resonances. The slope of the $J$-dependence of the gross structure of these resonances is consistent with an inertia parameter for two nearly touching ${ }^{12} \mathrm{C}$ nuclei, and many of the theoretical studies of these resonances seem to confirm the usefulness of the quasimolecular picture. One of the distinctive features of the resonances in the ${ }^{12} \mathrm{C}+{ }^{12} \mathrm{C}$ excitation functions ${ }^{2,3}$ ) is the rich fine structure of these resonances. As many as 6-10 closely spaced resonances of the same $J^{\pi}$ are observed in many different exit channels in a 2-3 $\mathrm{MeV}$ interval, individual fine structure peaks having widths of $100-200 \mathrm{keV}$. A com-

\footnotetext{
* Nishina Memorial Foundation Fellow, on leave of absence from Physics Department, Niigata University, Niigata 950-21, Japan.

+ Work supported by the US National Science Foundation.
} 
plete understanding of this fine structure, seemingly related to the nonspherical shape of the two ${ }^{12} \mathrm{C}$ nuclei, may serve as one of the most severe tests of the quasimolecular interpretation of these resonances.

Most recent theoretical interpretations are based on some variant of the Imanishi model ${ }^{4}$ ), yet despite extensive theoretical effort by the Greiner school ${ }^{5}$ ), Abe, Matsuse and Kondo ${ }^{6}$ ), and many others ${ }^{7}$ ), a detailed explanation of these resonances has not yet been given in the framework of a fully microscopic theory. A ${ }^{12} \mathrm{C}+{ }^{12} \mathrm{C}$ cluster model description of the $A=24$ system, possibly coupled to cluster states with fragment decompositions corresponding to the most important exit channels ${ }^{8}$ ), is the only model sufficiently microscopic for a detailed examination of this problem. In many simple cluster systems, e.g. the $\alpha+\alpha$ or $\alpha+{ }^{12} \mathrm{C}$ systems, the exclusion of Pauli-forbidden components in the wave functions is often more essential than the inclusion of the exchange terms in the interaction kernel, and the latter can be approximated by an effective local potential. Such systems have therefore been studied successfully in the framework of the orthogonality condition model $\left.(\mathrm{OCM})^{9}\right)$. In heavier systems the usefulness of the OCM is not established. However, in a system such as the ${ }^{12} \mathrm{C}+{ }^{12} \mathrm{C}$ "molecules", a cluster system made up of two nearly touching fragments, with fragment internal excitations limited mainly to rotational excitations such as the $0^{+}, 2^{+}, 4^{+}$ excitations of the ${ }^{12} \mathrm{C}$ fragments, the exclusion of Pauli-forbidden components may again be more important than an inclusion of the exchange terms in the interaction. In view of the great difficulty of a fully microscopic coupled-channel treatment of such a system, it may be worthwhile first to attempt a detailed OCM investigation. Recently, norm and overlap matrix elements for the binary fragment decompositions ${ }^{12} \mathrm{C}+{ }^{12} \mathrm{C}$, ${ }^{8} \mathrm{Be}+{ }^{16} \mathrm{O}, \alpha+{ }^{20} \mathrm{Ne}, \mathrm{p}+{ }^{23} \mathrm{Na}, \mathrm{n}+{ }^{23} \mathrm{Mg}$ of the $A=24$ system have been made available ${ }^{10}$ ), making it.possible to undertake such an investigation.

In a very preliminary study ${ }^{11}$ ) a phenomenological interaction with $Q \cdot Q$ terms was used to examine the question: is a simple ${ }^{12} \mathrm{C}+{ }^{12} \mathrm{C}$ molecular basis sufficient to account for the density of low- $J$ resonances in the Coulomb barrier region? For extreme simplicity only those components of the ${ }^{12} \mathrm{C}+{ }^{12} \mathrm{C}$ cluster model basis corresponding to $2 \hbar \omega$ shell-model excitations were retained in the basis, and for this reason this initial calculation was unable to predict (even approximately) the absolute positions of the resonances or make estimates of their widths. Instead, spectroscopic factors were used as a rough indicator of their strengths. In earlier OCM studies of the $A=24$ system, an ${ }^{16} \mathrm{O}+2 \alpha$ model ${ }^{12}$ ) was used to focus on the low-lying excitations in ${ }^{24} \mathrm{Mg}$. OCM calculations for uncoupled $\alpha+{ }^{20} \mathrm{Ne},{ }^{8} \mathrm{Be}+{ }^{16} \mathrm{O}$, and ${ }^{12} \mathrm{C}+{ }^{12} \mathrm{C}$ models ${ }^{13}$ ) gave an indication of the appearance of low- $J$ molecular states in the $22 \mathrm{MeV}$ excitation region, with large ${ }^{12} \mathrm{C}+{ }^{12} \mathrm{C}$ reduced width amplitudes. A first attempt to focus on the details of the fine structure of individual $J^{\pi}$ resonances has very recently been made in a pure ${ }^{12} \mathrm{C}+{ }^{12} \mathrm{C}$ basis ${ }^{14}$ ).

It is the purpose of the present investigation to generalize the earlier attempts with a cluster model basis sufficiently rich to reproduce the degree of complexity of the resonance fine structure and including a sufficient number of relative motion oscillator 
excitations to approach a semi-realistic description of the cluster radial wave functions. As in ref. ${ }^{11}$ ) the present study focuses on the low- $J$ Coulomb barrier region of the ${ }^{12} \mathrm{C}+{ }^{12} \mathrm{C}$ system since an interpretation in terms of quasibound states may be most appropriate for this region. The basis of our model is defined in sect. 2. Since a simple ${ }^{12} \mathrm{C}+{ }^{12} \mathrm{C}$ quasimolecular basis seems to be insufficient to account for all of the rich fine structure of the observed resonances ${ }^{6,11}$ ) the present model couples the ${ }^{12} \mathrm{C}+{ }^{12} \mathrm{C}$, $\alpha+{ }^{20} \mathrm{Ne}$, and ${ }^{8} \mathrm{Be}+{ }^{16} \mathrm{O}$ channels. Relative motion oscillator excitations have been limited to values corresponding to shell model excitations with oscillator excitation energies $\leqq 8 \hbar \omega$ to keep the dimension of the basis manageable. The details of the OCM equations of motion applied to this cluster model basis are presented in sect. 3. The cluster-cluster potential function is obtained from an effective $\mathrm{NN}$ interaction via a folding procedure in sect. 4 . This leads to a specific form for the radial dependence of the cluster relative motion potential function, including a spherically symmetric term and $Q \cdot Q$ couplings due to the nonspherical intrinsic shapes of ${ }^{12} \mathrm{C},{ }^{8} \mathrm{Be}$, and ${ }^{20} \mathrm{Ne}$ fragments. Although a small (10-20\%) adjustment of both the overall strength and diffuseness parameters of the folded potentials are introduced in the separate ${ }^{12} \mathrm{C}+{ }^{12} \mathrm{C}$, ${ }^{8} \mathrm{Be}+{ }^{16} \mathrm{O}$, and $\alpha+{ }^{20} \mathrm{Ne}$ channels to gain a consistent picture of the low-energy spectrum of the $A=24$ system, no further parameter adjustments are made in the $Q \cdot Q$ terms of the interaction which are responsible for the details of the fine structure of the resonances. The results of the present investigation are discussed in sect. 5. No attempt has been made to optimize the agreement between the model and experiment since the aim of this work is limited to showing the feasibility of a microscopic cluster model description of the resonances, including a coupling of the ${ }^{12} \mathrm{C}+{ }^{12} \mathrm{C}$ basis to the most important rearrangement channels. Although our basis is not expected to be rich enough to give a quantitative comparison between the theoretical model and experiment, it appears to be possible to identify a $5 \mathrm{MeV}$ region in the predicted spectrum as the seat of the candidates for the $J^{\pi}=0^{+}, 2^{+}, 4^{+}$molecular resonances. However, the fact that the predicted excitation energies are too high (by about $3 \mathrm{MeV}$ ) and the fact that the predicted ${ }^{12} \mathrm{C}+{ }^{12} \mathrm{C}$ reduced width amplitude in the surface region (with $R \gtrsim 5.5 \mathrm{fm}$ ) are too small and not sufficiently developed to be of true molecular character, shows that the basis of the present investigation must be expanded to include higher oscillator excitations in the relative motion degrees of freedom. It is nevertheless possible to conclude from this study that a microscopic cluster model picture can account for the major features of the observed resonances in the Coulomb barrier region of the ${ }^{12} \mathrm{C}+{ }^{12} \mathrm{C}$ system. It may, however, be more accurate to characterize the quasibound states responsible for the resonances as quasi-molecular rather than true molecular states, if a true molecular state is to be characterized by an extreme peaking of the ${ }^{12} \mathrm{C}+{ }^{12} \mathrm{C}$ relative motion function at an internuclear distance of $\sim 5.5 \mathrm{fm}$. 


\section{The cluster model basis}

Since a pure ${ }^{12} \mathrm{C}+{ }^{12} \mathrm{C}$ cluster model basis may not be enough to account for the full fine structure of the resonances, the $\alpha+{ }^{20} \mathrm{Ne}$ and ${ }^{8} \mathrm{Be}+{ }^{16} \mathrm{O}$ channels are to be coupled to the ${ }^{12} \mathrm{C}+{ }^{12} \mathrm{C}$ channel and are thus included explicitly in our microscopic cluster model basis. The cluster model functions are to be expanded in an SU(3)-coupled harmonic oscillator basis, with cluster-internal and cluster-relative-motion functions with equal oscillator width parameters. The norm and overlap diagonalizations are greatly simplified in such an SU(3)-coupled cluster basis. It therefore forms a natural basis for the OCM approximation. The properly antisymmetrized SU(3)-coupled cluster model functions are to be denoted by $\psi_{a}$,

$$
\psi_{\alpha}=\mathscr{A}\left|\left[\left[\phi^{\left(\lambda_{f} \mu_{f}\right)}(f) \times \phi^{\left(\boldsymbol{\lambda}_{1-f} \mu_{A-f}\right)}(A-f)\right]^{\left(\lambda_{q} \mu_{c}\right)} \times \chi^{(\mathbf{Q} 0)}\left(\boldsymbol{R}_{f}\right)\right]_{\kappa J M}^{(\lambda \mu)}\right\rangle,
$$

where $\mathscr{A}$ antisymmetrizes $\psi_{\alpha}$ under exchange of nucleons between cluster fragments of mass $f$ and $A-f$. The square brackets denote SU(3)-coupling. [The notation of the paper follows that of ref. ${ }^{10}$ ), particularly insofar as the SU(3) quantum numbers and coupling coefficients are concerned.] In the above, $\alpha$ stands for the quantum numbers $\left(\lambda_{c} \mu_{c}\right),(\lambda \mu), \kappa$ and specifies the nature of the fragments $f, A-f$. Specifically, the $\psi_{\alpha}$ include:

(i) the ${ }^{12} \mathrm{C}+{ }^{12} \mathrm{C}$ basis

$$
\mathscr{A}\left|\left[\left[\phi^{(04)}\left({ }^{12} \mathrm{C}\right) \times \phi^{(04)}\left({ }^{12} \mathrm{C}\right)\right]^{\left(\lambda_{\tau} \mu_{c}\right)} \times \chi^{(00)}\left(\boldsymbol{R}_{12-12}\right)\right]_{\kappa J M}^{(\lambda \mu)}\right\rangle,
$$

(ii) the $\alpha+{ }^{20} \mathrm{Ne}$ basis

$$
\mathscr{A}\left|\left[\left[\phi^{(00)}(\alpha) \times \phi^{(80)}\left({ }^{20} \mathrm{Ne}\right)\right]^{(80)} \times \chi^{(Q-4,0)}\left(\boldsymbol{R}_{4-20}\right)\right]_{\kappa J M}^{(\lambda \mu)}\right\rangle,
$$

(iii) the ${ }^{8} \mathrm{Be}+{ }^{16} \mathrm{O}$ basis

$$
\mathscr{A}\left|\left[\left[\phi^{(40)}\left({ }^{8} \mathrm{Be}\right) \times \phi^{(00)}\left({ }^{16} \mathrm{O}\right)\right]^{(40)} \times \chi^{(00)}\left(R_{8-16}\right)\right]_{\kappa J M}^{(\lambda \mu)}\right\rangle .
$$

The fragment internal wave functions, $\phi$, are the simplest possible shell-model wave functions of pure $\mathrm{SU}(3)$ symmetry; e.g., $\left(\lambda_{f} \mu_{f}\right)=(04)$ for $f={ }^{12} \mathrm{C}$. The basis thus includes the excitation of each ${ }^{12} \mathrm{C}$ fragment to $2^{+}$and $4^{+}$states, and similarly the excitation of ${ }^{20} \mathrm{Ne}$ to $2^{+}, 4^{+}, 6^{+}, 8^{+}$states and ${ }^{8} \mathrm{Be}$ to $2^{+}, 4^{+}$states, that is all members of the ground-state rotational band of each cluster fragment. The relative motion harmonic oscillator wave function $\chi^{\left(Q^{0)}\right.}\left(\boldsymbol{R}_{f}\right)$ carries $Q$ oscillator quanta; $\boldsymbol{R}_{f}$ is the appropriate dimensionless relative coordinate for fragments $f$ and $A-f$ :

$$
\boldsymbol{R}_{f}=\frac{1}{b}\left\{\left[\frac{A-f}{A f}\right]^{\sharp}\left(\boldsymbol{r}_{1}+\ldots+\boldsymbol{r}_{f}\right)-\left[\frac{f}{A(A-f)}\right]^{\ddagger}\left(\boldsymbol{r}_{f+1}+\ldots+\boldsymbol{r}_{A}\right)\right\},
$$


where $b=[h / m \omega]^{\ddagger}, m=$ nucleon mass. Since we are interested in positive-parity states, $Q$ must be even, and in the ${ }^{12} \mathrm{C}+{ }^{12} \mathrm{C}$ channel $\left(\lambda_{c} \mu_{c}\right)$ is restricted to (08), (24) and (40).

The smallest Pauli-allowed value of $Q$ is 12 . For $Q=12$ the Pauli-allowed states are restricted to those with $(\lambda \mu)=(84),(73),(62),(51),(40)$ in the ${ }^{12} \mathrm{C}+{ }^{12} \mathrm{C}$ channel, $(\lambda \mu)=(84),(46),(08)$ in the $\alpha+{ }^{20} \mathrm{Ne}$ channel, and $(\lambda \mu)=(84)$ in the ${ }^{8} \mathrm{Be}+{ }^{16} \mathrm{O}$ channel. Note, however, that the three (84) states are identically equal, since there is but a single shell-model state with $(\lambda \mu)=(84)$. It is the dominant component of the ground rotational band of ${ }^{24} \mathrm{Mg}$. To eliminate redundant states and form an orthogonal basis of independent Pauli-allowed states, the norm and overlap matrix diagonalization is carried out:

$$
\sum_{\alpha^{\prime}}\left\langle\psi_{a} \mid \psi_{a^{\prime}}\right\rangle c_{a^{\prime}}=\beta c_{a^{*}}
$$

In the SU(3)-coupled basis, $\psi_{\alpha}$, the overlap matrix elements $\left\langle\psi_{a} \mid \psi_{\alpha^{\prime}}\right\rangle$ are diagonal in $(\lambda \mu)$ and $\kappa$, and independent of $\kappa J M$. The diagonalization is therefore factored and can be carried out separately in each $(\lambda \mu)$ subspace. For each such $(\lambda \mu)$ subspace the summation in eq. (4) thus runs only over the fragment designations and the quantum numbers $\left(\lambda_{c} \mu_{c}\right)$ (when needed). The sums over $\alpha^{\prime}$ thus contain at most five terms in our chosen basis. With $\sum_{\alpha^{\prime}}\left|c_{\alpha^{\prime}}\right|^{2}=1$, eq. (4) leads to the orthonormal basis

$$
\left\{\sqrt{\frac{1}{\beta}} \sum_{\alpha} c_{\alpha} \psi_{\alpha}\right\}
$$

where the states, (5), are eigenstates of the antisymmetrizer in the chosen Hilbert space.

Table 1 lists the SU(3) quantum numbers, $(\lambda \mu)$, of the Pauli-allowed states $(\beta \neq 0)$ both for the restricted basis of a pure ${ }^{12} \mathrm{C}+{ }^{12} \mathrm{C}$ channel (table 1a) and for the full basis

TABI.E la

\begin{tabular}{|c|c|}
\hline$Q$ & $(\lambda \mu)^{n}$ \\
\hline 12 & $(84),(73),(62),(51),(40)$ \\
\hline 14 & $(14,2),(11,5),(12,3),(13,1),(10,4)^{2},(11,2),(12,0),(93)^{2},(10,1),(82)^{2},(71),(60)$ \\
\hline 16 & $\begin{array}{l}(20,0),(17,3),(18,1),(14,6),(15,4),(16,2)^{2},(13,5)^{2},(14,3)^{2},(15,1),(12,4)^{3},(13,2) \\
(14,0),(11,3)^{2},(12,1),(10,2)^{2},(91),(80)\end{array}$ \\
\hline 18 & $\begin{array}{l}(20,4),(22,0),(17,7),(18,5),(19,3),(20,1),(16,6)^{2},(17,4),(18,2)^{2},(15,5)^{2},(16,3)^{2} \\
(17,1),(14,4)^{3},(15,2),(16,0),(13,3)^{2},(14,1),(12,2)^{2},(11,1),(10,0)\end{array}$ \\
\hline 20 & $\begin{array}{l}(20,8),(22,4),(24,0),(19,7),(20,5),(21,3),(22,1),(18,6)^{2},(19,4),(20,2)^{2},(17,5)^{2} \\
(18,3)^{2},(19,1),(16,4)^{3},(17,2),(18,0),(15,3)^{2},(16,1),(14,2)^{2},(13,1),(12,0)\end{array}$ \\
\hline
\end{tabular}

Pauli-allowed states in the ${ }^{12} \mathrm{C}+{ }^{12} \mathrm{C}$ basis 
TABLE lb

Pauli-allowed states in the ${ }^{12} \mathrm{C}+{ }^{12} \mathrm{C}, x+{ }^{20} \mathrm{Ne},{ }^{8} \mathrm{Be}+{ }^{16} \mathrm{O}$ coupled basis

\begin{tabular}{|c|c|}
\hline$Q$ & $(\lambda \mu)^{n}$ \\
\hline 12 & $(84),(73),(46),(08),(62),(51),(40)$ \\
\hline 14 & $\begin{array}{l}(14,2),(11,5),(12,3)^{2},(13,1),(10,4)^{4},(11,2),(12,0),(85),(93)^{2},(10,1),(66),(47),(82)^{2}, \\
(28),(71),(60)\end{array}$ \\
\hline 16 & $\begin{array}{l}(20,0),(17,3),(18,1)^{2},(14,6),(15,4),(16,2)^{4},(13,5)^{2},(14,3)^{4},(15,1),(12,4)^{5},(13,2), \\
(14,0),(10,5),(11,3)^{2},(12,1),(86),(67),(10,2)^{2},(48),(91),(80)\end{array}$ \\
\hline 18 & $\begin{array}{l}(20,4),(22,0)^{3},(17,7),(18,5),(19,3),(20,1)^{3},(16,6)^{2},(17,4),(18,2)^{4},(15,5)^{2},(16,3)^{4}, \\
(17,1),(14,4)^{5},(15,2),(16,0),(12,5),(13,3)^{2},(14,1),(10,6) ;(12,2)^{2},(87),(68),(11,1), \\
(10,0)\end{array}$ \\
\hline 20 & $\begin{array}{l}(20,8),(22,4),(24,0)^{3},(19,7),(20,5),(21,3),(22,1)^{3},(18,6)^{2},(19,4),(20,2)^{4},(17,5)^{2}, \\
(18,3)^{4},(19,1),(16,4)^{3},(17,2),(18,0),(14,5),(15,3)^{2},(16,1),(12,6),(14,2)^{2},(10,7),(88), \\
(13,1),(12,0)\end{array}$ \\
\hline
\end{tabular}

of all three channels (table $1 \mathrm{~b}$ ). For $Q \geqq 20$ the possible $(\lambda \mu)$ states are all linearly independent and become allowed. The rich structure of many possible states in the ${ }^{12} \mathrm{C}+{ }^{12} \mathrm{C}$ basis may explain why many fine structure components are observed in the resonances of the ${ }^{12} \mathrm{C}+{ }^{12} \mathrm{C}$ excitation functions. Some representations, $(\lambda \mu)$, appear in all three channels, in particular $(\lambda \mu)=(Q+4,0)(Q+2,1)(Q, 2)(Q-2,3)(Q-4,4)$. Overlap matrix elements in these states are significantly large. As an example, the overlap matrix elements for states with $Q=16,(\lambda \mu)=(12,4)$ are shown in table 2 . Such large overlaps are consistent with the fact that many Coulomb barrier resonances observed in the ${ }^{12} \mathrm{C}+{ }^{12} \mathrm{C}$ induced reaction are strongly correlated in various exit channels of $\alpha+{ }^{20} \mathrm{Ne}$ and ${ }^{8} \mathrm{Be}+{ }^{16} \mathrm{O}$.

The coupling of ${ }^{12} \mathrm{C}+{ }^{12} \mathrm{C}, \alpha+{ }^{20} \mathrm{Ne}$, and ${ }^{8} \mathrm{Be}+{ }^{16} \mathrm{O}$ channels appears to be important for a proper treatment of the Coulomb-barrier resonance region. The nucleon

TABle 2

Overlaps for states with $Q=16,(\lambda \mu)=(12,4)$

\begin{tabular}{cccccc}
\hline $\begin{array}{c}f+(A-f) \\
\left(\lambda_{r} \mu_{c}\right)\end{array}$ & $\begin{array}{c}{ }^{12} \mathrm{C}+{ }^{12} \mathrm{C} \\
(08)\end{array}$ & $\begin{array}{c}{ }^{12} \mathrm{C}+{ }^{12} \mathrm{C} \\
(24)\end{array}$ & $\begin{array}{c}{ }^{12} \mathrm{C}+{ }^{12} \mathrm{C} \\
(40)\end{array}$ & $\begin{array}{c}\alpha+{ }^{20} \mathrm{Ne} \\
(80)\end{array}$ & $\begin{array}{c}{ }^{8} \mathrm{Be}+{ }^{16} \mathrm{O} \\
(40)\end{array}$ \\
\hline & $\left.1.0^{2}\right)$ & 0.303870 & 0.129501 & 0.185016 & 0.492974 \\
& 1.0 & 0.661192 & 0.177461 & 0.564195 \\
& & 1.0 & 0.125391 & 0.413667 \\
& & & 1.0 & 0.256305 \\
& & & & 1.0 \\
\hline
\end{tabular}

-) To focus on the magnitude of the overlaps the states $\psi_{a}$ have been renormalized to give diagonal entries of 1.0. The actual values of the norms are $0.403145,0.222080,0.0850768,0.674438$ and 0.386503 . 
channels have been neglected largely for simplicity even though some ${ }^{12} \mathrm{C}+{ }^{12} \mathrm{C}$ basis states have significant overlaps with the $\mathrm{p}+{ }^{23} \mathrm{Na}$ and $\mathrm{n}+{ }^{23} \mathrm{Mg}$ channels $\left.{ }^{10}\right)$. It may perhaps be expected that the nucleon channels do not play a major role in the dynamics of the resonances, that is in the determination of the nature of the quasibound states, although they must be taken into account in a quantitative determination of the widths. Even without the nucleon channels the microscopic cluster model basis of the present investigation is a rich one. With $Q$ restricted to $12 \leqq Q \leqq 20$ the dimensions of the bases for $J=0,2$, and 4 are 48,137 , and 190 in the restricted space of the pure ${ }^{12} \mathrm{C}+{ }^{12} \mathrm{C}$ channel. These are increased to 76,208 , and 292 for the full basis of mixed ${ }^{12} \mathrm{C}+{ }^{12} \mathrm{C}$, $\alpha+{ }^{20} \mathrm{Ne}$, and ${ }^{8} \mathrm{Be}+{ }^{16} \mathrm{O}$ channels.

\section{The OCM equation of motion}

The orthogonality condition model (OCM) approximation was first introduced in the $\alpha-\alpha$ scattering problem ${ }^{9}$ ) since it accounts well for the almost energy-independent inner oscillatory behavior of the $\alpha-\alpha$ scattering wave function of RGM type. The OCM has been applied successfully to describe the spectrum of ${ }^{16} \mathrm{O}$ below $\sim 15 \mathrm{MeV}$ through an $\alpha+{ }^{12} \mathrm{C}$ cluster model ${ }^{15}$ ) and gives a good account of the scattering ${ }^{16}$ ) of $\alpha$-particles on ${ }^{12} \mathrm{C}$. Recently, the structure of ${ }^{20} \mathrm{Ne}$ has been investigated with the use of a coupled $\left(\alpha+{ }^{16} \mathrm{O}\right),\left({ }^{8} \mathrm{Be}+{ }^{12} \mathrm{C}\right)$ treatment $\left.{ }^{17}\right)$. Although progress has been made in the evaluation of the full interaction kernel for the ${ }^{12} \mathrm{C}+{ }^{12} \mathrm{C}, \alpha+{ }^{20} \mathrm{Ne},{ }^{8} \mathrm{Be}+{ }^{16} \mathrm{O}$ cluster decomposition of the $A=24$ system ${ }^{18}$ ), a fully microscopic multichannel RGM or GCM treatment of the ${ }^{12} \mathrm{C}+{ }^{12} \mathrm{C}$ excitation functions would constitute a major computational effort. If exchange terms in the interaction kernel do not play a prominent role in the resonant structure of the ${ }^{12} \mathrm{C}+{ }^{12} \mathrm{C}$ system, an OCM treatment in which Pauli-forbidden components are properly excluded from the basis, even though exchange terms in the interaction are not treated correctly, may form a more reasonable starting point for a microscopic study of this problem. The availability of the needed norm and overlap kernels makes such a calculation tractable.

In the OCM approximation, the hamiltonian, $H$, is approximated by $\mathscr{H}$ where

$$
\mathscr{H}=\sqrt{K}\left[T+V+H_{\text {internal }}\right] \sqrt{K} .
$$

In this effective $\mathscr{H}, K$ is the overlap kernel which insures the exclusion of Pauliforbidden components, $T$ is the kinetic energy operator for the relative motion of fragments $f$ and $A-f, V$ is an effective local potential between clusters, and $H_{\text {internal }}$ gives the internal excitation energy of the fragments.

To make the meaning of the operator $K$ more precise, it is useful to discuss first the transformation from the SU(3)-coupled cluster model basis, $\psi_{\alpha}$ [see eq. (2)], to the more conventional channel spin basis,

$$
\psi_{i}=\mathscr{A}\left|\left(\left(\phi_{I_{f}}^{\left(\lambda_{f} \mu_{f}\right)}(f) \times \phi_{I_{A-f}}^{\left(\lambda_{A-f} \mu_{A-f)}\right.}(A-f)\right)_{I_{c}} \times \chi_{L}^{(Q 0)}\left(R_{f}\right)\right)_{S_{M}}\right\rangle,
$$


where $i$ denotes the angular momentum quantum numbers, $\left(I_{f} I_{A}-f_{c} L\right)$ of the channel spin scheme. Note that the label $i$ also includes the fragment designation $f$ (and hence $A-f)$. In eq. (7) the round brackets denote ordinary angular momentum coupling. The $\psi_{i}$ and $\psi_{\alpha}$ are related by the orthogonal transformation

$$
\psi_{i}=\sum_{\alpha} U_{i a} \psi_{a}, \quad \psi_{a}=\sum_{i} U_{i a} \psi_{i}
$$

where

$$
\begin{aligned}
U_{l \alpha}= & \sum_{\kappa_{c}}\left\langle\left(\lambda_{f} \mu_{f}\right) I_{f} ;\left(\lambda_{A-f} \mu_{A-f}\right) I_{A-f} \|\left(\lambda_{c} \mu_{c}\right) \kappa_{c} I_{c}\right\rangle \\
& \times\left\langle\left(\lambda_{c} \mu_{c}\right) \kappa_{c} I_{c} ;(Q 0) L \|(\lambda \mu) \kappa J\right\rangle .
\end{aligned}
$$

Since these $U_{i a}$ are real,

$$
\sum_{\alpha} U_{i \alpha} U_{j \alpha}=\delta_{i j} \quad \sum_{i} U_{i \alpha} U_{i \beta}=\delta_{\alpha \beta}
$$

Note that both labels $\alpha$ and $i$ include the cluster designation $f$; but the transformation coefficients are diagonal in the fragment decomposition and do not connect states with $f^{\prime} \neq f$. Thus the sum over $\alpha$ (or $i$ ) includes only sums over the labels $(\lambda \mu), \kappa$, and $\left(\lambda_{c} \mu_{c}\right)$ (or $I_{I_{A}} I_{A-f} I_{c} L$ ) appropriate for the fixed fragment decomposition implied by $i$ (or $\alpha$ ). If the relative motion functions are factored into radial and angular parts

$$
\chi_{L M}^{\left(Q^{00}\right)}\left(\boldsymbol{R}_{f}\right)=\mathscr{X}_{L}^{(0)}\left(R_{f}\right) Y_{L M}\left(\hat{R}_{f}\right)
$$

where $\hat{\boldsymbol{R}}_{\boldsymbol{f}}$ denotes a unit vector, the overlap kernel can be defined through

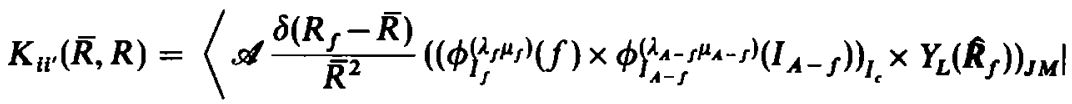

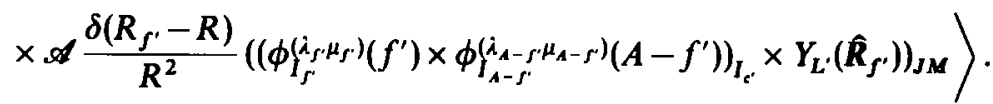

The norm and overlap matrix elements are related to the matrix elements of $K$ by

$$
\begin{aligned}
\left\langle\psi_{\alpha} \mid \psi_{\alpha^{\prime}}\right\rangle & =\sum_{i i^{\prime}} U_{i \alpha} U_{i^{\prime} \alpha^{\prime}}\left\langle\mathscr{R}_{L}^{(Q 0)}\left|K_{i i^{\prime}}\right| \mathscr{R}_{L^{\prime}}^{\left(Q^{\prime} 0\right)}\right\rangle \\
& =\sum_{i i^{\prime}} U_{i \alpha} U_{i^{\prime} \alpha^{\prime}} \int \mathrm{d} \bar{R} \bar{R}^{2} \int \mathrm{d} R R^{2} \mathscr{R}_{L}^{(Q 0)}(\bar{R})^{*} K_{i l^{\prime}}(\bar{R}, R) \mathscr{R}_{L}^{\left(Q^{\prime} 0\right)}(R) .
\end{aligned}
$$

Also,

$$
\sum_{i^{\prime}} K_{i i^{\prime}}\left(\sum_{\alpha^{\prime}} c_{\alpha^{\prime}} U_{i^{\prime} \alpha^{\prime}} \mathscr{R}_{L^{\prime}}^{\left(Q^{\prime} 0\right)}\right)=\beta\left(\sum_{\alpha} c_{\alpha} U_{i \alpha} \mathscr{R}_{L^{(0)}}^{(Q 0)}\right),
$$


namely,

$$
\begin{aligned}
\sum_{i^{\prime}} \sum_{\alpha^{\prime}} c_{\alpha^{\prime}} U_{i^{\prime} \alpha^{\prime}} \int \mathrm{d} R R^{2} K_{i i^{\prime}}(\bar{R}, R) \mathscr{R}_{L}^{\left(Q^{\prime} 0\right)}(R) \\
=\beta\left(\sum_{\alpha} c_{\alpha} U_{i \alpha^{\prime}} \Re_{L}^{(Q 0)}(\bar{R})\right),
\end{aligned}
$$

where eq. (14) is the channel spin analog of eq. (4). In eq. (14) the sum over $i^{\prime}$ (and hence $\alpha^{\prime}$ ) includes a summation over all fragment decompositions $f$, whereas the index $i$ stands for channel spin quantum numbers for a fixed $f$.

In the OCM approximation the hamiltonian matrix elements between the orthonormalized cluster model basis states

$$
\left\langle\sqrt{\frac{1}{\beta}} \sum_{\alpha} c_{\alpha} \psi_{\alpha}|H| \sqrt{\frac{1}{\beta^{\prime}}} \sum_{\alpha^{\prime}} c_{\alpha^{\prime}} \psi_{\alpha^{\prime}}\right\rangle
$$

are replaced by

$$
\begin{aligned}
& \sqrt{\frac{1}{\beta \beta^{\prime}}} \sum_{\alpha \alpha^{\prime}} \sum_{i i^{\prime}} c_{\alpha} c_{\alpha^{\prime}} U_{i \alpha} U_{i^{\prime} \alpha^{\prime}}\left\langle\Re_{L}^{(Q 0)}\left|\mathscr{H}_{i i^{\prime}}\right| \mathscr{R}_{L^{\prime}}^{\left(Q^{\prime}\right)}\right\rangle \\
& =\sum_{a a^{\prime}} \sum_{i} c_{\alpha} U_{i \alpha} \sum_{i^{\prime}} c_{x^{\prime}} U_{l^{\prime} x^{\prime}}\left\langle\mathscr{N}_{L}^{(Q 0)}\left|\left[T+V+H_{\text {interna }}\right]_{i i^{\prime}}\right| \mathbb{R}_{L^{\prime}}^{\left(Q^{\prime} 0\right)}\right\rangle \\
& =\sum_{\alpha a^{\prime}} c_{\alpha} c_{\alpha^{\prime}}\left\langle\bar{\psi}_{\alpha}\left|T+V+H_{\text {internal }}\right| \bar{\psi}_{a^{\prime}}\right\rangle
\end{aligned}
$$

In the last form of eq. (15b) the functions $\bar{\psi}_{x}$ do not include the antisymmetrizer $\mathscr{A}$ (which has been eliminated by the operation of $\sqrt{ } K$ ); that is $\psi_{\alpha}$ is defined by $\psi_{\alpha}=\mathscr{A} \psi_{x}$.

In terms of such SU(3)-coupled cluster functions, $\bar{\psi}_{x}$, the matrix elements of the operators $\mathcal{O}$, where $\mathcal{O}$ is $T$, or $V$, or $H_{\text {internal }}$ are first related to matrix elements $M_{\mathcal{O}}$. The latter are defined by

$$
\begin{aligned}
& \left\langle\left[\left[\phi^{\left(\lambda_{f} \mu_{f}\right)}(f) \times \phi^{\left(\lambda_{1-f} \mu_{A-f}\right)}(A-f)\right]^{\left(\lambda_{c} \mu_{c}\right)} \times \chi^{(Q 0)}\left(R_{f}\right)\right]_{\kappa J M}^{(\lambda \mu)}\right| \mathcal{O} \\
& \left|\left[\left[\phi^{\left(\lambda_{f} \cdot \mu_{f}\right)}\left(f^{\prime}\right) \times \phi^{\left(\lambda_{A-f^{\prime}} \mu_{A-f^{\prime}}\right)}\left(A-f^{\prime}\right)\right]^{\left(\lambda_{r^{\prime}} \mu_{c^{\prime}}\right)} \times \chi^{\left(Q^{\prime} 0\right)}\left(\boldsymbol{R}_{f^{\prime}}\right)\right]_{\kappa^{\prime} J M}^{\left(\lambda^{\prime} \mu^{\prime}\right)}\right\rangle \\
& =\sum_{\kappa_{e} I_{c} L} \sum_{\kappa c, I c, L^{\prime}}\left\langle\left(\lambda_{c} \mu_{c}\right) \kappa_{c} I_{c} ;(Q 0) L \|(\lambda \mu) \kappa J\right\rangle \\
& \times\left\langle\left(\hat{\lambda}_{c^{\prime}} \mu_{c^{\prime}}\right) \kappa_{c^{\prime}} I_{c^{\prime}} ;\left(Q^{\prime} 0\right) L^{\prime} \|\left(\lambda^{\prime} \mu^{\prime}\right) \kappa^{\prime} J\right\rangle M_{\Theta}
\end{aligned}
$$

where

$$
\begin{aligned}
& M_{\mathcal{O}}=\left\langle\left(\left[\phi^{\left(\lambda_{f} \mu_{f}\right)}(f) \times \phi^{\left(\lambda_{1-f} \mu_{A-f}\right)}(A-f)\right]_{\kappa_{c} I_{c}}^{\left(\lambda_{c} \mu_{c}\right)} \times \chi_{L}^{(\ell 0)}\left(\boldsymbol{R}_{f}\right)\right)_{J M}\right|
\end{aligned}
$$

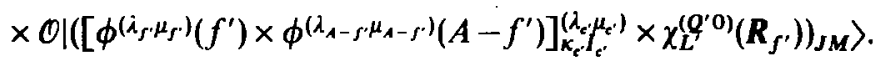


For $\mathcal{O}=T$ or $\mathcal{O}=H_{\text {internal, }}$ the $M_{\mathcal{O}}$ are very simple:

(i) The kinetic energy. With $\mathcal{O}=T$,

$$
\begin{aligned}
M_{T}= & \delta_{f, f^{\prime}} \delta_{\left(\lambda_{\tau} \mu_{c}\right) \kappa_{d_{c}}\left(\lambda_{c^{\prime}} \mu_{c^{\prime}}\right) \kappa_{c^{\prime}} I_{c^{\prime}}} \delta_{L, L^{\prime}} \\
& \times \frac{1}{4} \hbar \omega\left\{\delta_{Q, Q^{\prime}}(2 Q+3)-\delta_{Q, Q^{\prime}+2}\left[\left(Q^{\prime}+L+3\right)\left(Q^{\prime}-L+2\right)\right]^{\frac{1}{t}}\right. \\
& \left.-\delta_{Q^{\prime}, Q+2}[(Q+L+3)(Q-L+2)]^{\frac{1}{2}}\right\}
\end{aligned}
$$

(ii) The cluster-internal energy. With $\mathcal{O}=H_{\text {internal, }}$,

$$
\begin{aligned}
M_{H_{\text {ideormat }}}= & \delta_{f, f^{\prime}} \delta_{I_{c}, I_{c^{\prime}}} \delta_{Q, Q^{\prime}} \delta_{L, L^{\prime}} \sum_{I_{f} I_{A-f}} \\
& \times\left\langle\left(\lambda_{f} \mu_{f}\right) I_{f} ;\left(\lambda_{A-f} \mu_{A-f}\right) I_{A-f} \|\left(\lambda_{c} \mu_{c}\right) \kappa_{c} I_{c}\right\rangle \\
& \times\left\langle\left(\lambda_{f} \mu_{f}\right) I_{f} ;\left(\lambda_{A-f} \mu_{A-f}\right) I_{A-f} \|\left(\lambda_{c^{\prime}} \mu_{c^{\prime}}\right) \kappa_{c^{\prime}} I_{c}\right\rangle \\
& \times\left\{E_{f}\left(I_{f}\right)+E_{A-f}\left(I_{A-f}\right)+\delta_{\left(\lambda_{d} \mu_{c}\right) \kappa_{c}\left(\lambda_{c}, \mu_{c}\right) \kappa_{c^{\prime}}} E_{\mathrm{th}}(f, A-f)\right\},
\end{aligned}
$$

where the energy is given relative to the ground state of ${ }^{24} \mathrm{Mg}$. The $E_{f}\left(I_{f}\right)$ are the excitation energies of the state with spin $I_{f}$ in nucleus $f$, and $E_{\mathrm{th}}(f, A-f)$ is the threshold energy for the decay to the $f+(A-f)$ channel.

Before proceeding to $M_{V}$ the choice of $V$ has to be discussed.

\section{The cluster-cluster potential function}

As in many other applications of the OCM, the effective local potential between clusters, $V$, is chosen to be a folded potential of a simple effective NN interaction. As in the coupled channel OCM study of ${ }^{20} \mathrm{Ne}\left[\right.$ ref. ${ }^{17}$ )], we also assume that the folded potential itself does not connect the different channels, and that the coupling arises only through the operator $K$.

The folded potential has been evaluated by the method of Goto and Horiuchi ${ }^{19}$ ) which is very well suited to the present case, where the cluster fragments are described by non-spherical internal states of good SU(3) symmetry. The NN interaction is assumed to be made up of a linear combination of terms of gaussian form:

$$
v\left(r_{i j}\right)=\exp \left[-\rho r_{i j}^{2}\right]\left(W+B P_{\sigma}-H P_{\tau}-M P_{\sigma} P_{\tau}\right),
$$

where $r_{i j}$ is the distance between nucleon $i$ in fragment $f$ and nucleon $j$ in fragment $A-f$. For such a gaussian term in the interaction the general form of the folded potential for 
fragments $f+A-f$ such as ${ }^{12} \mathrm{C}+{ }^{12} \mathrm{C}, \alpha+{ }^{20} \mathrm{Ne}$, and ${ }^{8} \mathrm{Be}+{ }^{16} \mathrm{O}$, is given by

$$
\begin{aligned}
V= & \frac{1}{16}(16 W+8 B-8 H-4 M)\left[\frac{\eta}{2 b^{2} \rho}\right]^{\frac{3}{3}} \exp \left[-\chi R^{2}\right] \\
& \times\left\{a_{1}+a_{2} \chi R^{2}+a_{3} \chi^{2} R\right. \\
& +\frac{16}{15} \pi\left(b_{1}+b_{2} \chi R^{2}\right)([(A-f) Q(f)+f Q(A-f)] \cdot Q(R)) \\
& \left.+\frac{16}{15} \pi \eta^{2}\left(\frac{1}{4}-\frac{1}{3} \chi R^{2}+\frac{1}{15} \chi^{2} R^{4}\right)(Q(f) \cdot Q(A-f))\right\} \\
& +\frac{Z_{f} Z_{A-f^{2}}}{[A / f(A-f)]^{\frac{1}{3}} b R} \operatorname{erf}\left(\frac{3 \sqrt{\pi}}{4 R_{0}} R\right),
\end{aligned}
$$

where

$$
\eta^{-1}=1-\frac{1}{2} \frac{A}{f(A-f)}+\frac{1}{2 b^{2} \rho}, \quad \chi=\frac{1}{2} \frac{A}{f(A-f)} \eta,
$$

and $R_{0}=r_{0}\left[f^{\ddagger}+(A-f)^{\ddagger}\right] / b[A / f(A-f)]^{\frac{1}{t}}$; with $r_{0}=1.3 \mathrm{fm}$. The coefficients $a_{i}, b_{i}$ are listed in table 3. The distance between fragments $f$ and $A-f$ is given in terms of the dimensionless relative coordinate, $R$, between fragments $f$ and $A-f$, defined in eq. (3); $b=[h / m \omega]^{t}$. The first term in the potential is a spherically symmetric potential. Due to the intrinsic non-spherical nature of the ${ }^{12} \mathrm{C},{ }^{8} \mathrm{Be}$, and ${ }^{20} \mathrm{Ne}$ wave functions of our basis, the folding procedure leads to quadrupole-quadrupole interaction terms as well. These $Q \cdot Q$ couplings are of two types, the first an interaction between internal and relative motion quadrupole terms, the second a $Q \cdot Q$ interaction of the internal-internal type which occurs only in the ${ }^{12} \mathrm{C}+{ }^{12} \mathrm{C}$ decomposition of our basis. The relative motion quadrupole operator is defined by $Q(R)=R^{2} Y_{2}(\hat{R})$. The quadrupole operators $Q(f)$, $Q(A-f)$ involving the cluster internal degrees of freedom are effectively Elliott-type $Q$ operators. (The normalization is such that $\frac{16}{15} \pi Q(f) \cdot Q(f)=C\left(\lambda_{f} \mu_{f}\right)-I_{f}\left(I_{f}+1\right)$, with

\begin{tabular}{|c|c|c|c|c|c|}
\hline$f+(A-f)$ & $a_{1}$ & $a_{2}$ & $a_{3}$ & $b_{1}$ & $b_{2}$ \\
\hline $\begin{array}{l}{ }^{12} \mathrm{C}+{ }^{12} \mathrm{C} \\
\alpha+{ }^{20} \mathrm{Ne} \\
{ }^{8} \mathrm{Be}+{ }^{16} \mathrm{O}\end{array}$ & $\begin{array}{c}144-96 \eta+\frac{80}{3} \eta^{2} \\
80-40 \eta+\frac{46}{7} \eta^{2} \\
128-80 \eta+20 \eta^{2}\end{array}$ & $\begin{array}{l}64 \eta-\frac{320}{9} \eta^{2} \\
\frac{80}{3} \eta-\frac{184}{21} \eta^{2} \\
\frac{160}{3} \eta-\frac{80}{3} \eta^{2}\end{array}$ & $\begin{array}{l}\frac{64}{64} \eta^{2} \\
\frac{18}{105} \eta^{2} \\
\frac{16}{3} \eta^{2}\end{array}$ & $\begin{array}{l}\left(\frac{1}{2}-\frac{7}{18} \eta\right) \eta \chi \\
\left(\frac{1}{2}+\frac{133}{160} \eta\right) \eta \chi \\
\left(\frac{1}{2}-\frac{7}{16} \eta\right) \eta \chi\end{array}$ & $\begin{array}{c}\frac{1}{9} \eta^{2} x \\
-\frac{18}{80} \eta^{2} x \\
\frac{1}{8} \eta^{2} x\end{array}$ \\
\hline
\end{tabular}
$C(\lambda \mu)=\frac{4}{3}\left(\lambda^{2}+\mu^{2}+\lambda \mu+3 \lambda+3 \mu\right)$.) Note that both the spherical and the $Q \cdot Q$ terms of the potential have, besides a polynomial dependence, a gaussian fall-off with increase in the relative motion variable $R$. A uniform-sphere Coulomb term is included via the

TABLE 3

Coefficients of the folded potentials 
error function approximation. It has been shown ${ }^{19}$ ) that the folded potentials for the ${ }^{12} \mathrm{C}+{ }^{12} \mathrm{C}$ and $\alpha+{ }^{20} \mathrm{Ne}$ channels include additional terms (of higher multipole character). These have been neglected since they are expected to make only minor contributions.

The spherical terms (that is the first and fourth terms) of $V$ make the following contributions to $M_{V}$ :

$$
\begin{aligned}
& M_{V}=\delta_{f, f}, \delta_{\left(\lambda_{c} \mu_{e}\right) \kappa_{e} l_{g}\left(\lambda_{c^{\prime}} \mu_{e}\right) \kappa_{e} l_{e^{\prime}}} \delta_{L, L^{\prime}} \\
& \times\left\{\frac{1}{16}(16 W+8 B-8 H-4 M)\left[\frac{\eta}{2 b^{2} \rho}\right]^{3}\left\langle\mathfrak{Q}_{L}^{(Q 0)}\right|\left(a_{1}+a_{2} \chi R^{2}+a_{3} \chi^{2} R^{4}\right)\right. \\
& \times \exp \left[-\chi R^{2}\right]\left|\mathscr{A}_{L}^{\left(Q^{\prime}\right)}\right\rangle \\
& \left.+\frac{Z_{f} Z_{A-f^{2}}}{[A / f(A-f)]^{\frac{1}{2} b}}\left\langle\mathscr{x}_{\mathrm{L}}^{(0)}\left|\frac{1}{R} \operatorname{erf}\left(\frac{3 \sqrt{\pi}}{4 R_{0}} R\right)\right| \mathscr{R}_{L}^{\left(Q^{\prime} 0\right)}\right\rangle\right\} .
\end{aligned}
$$

The contribution of the second (internal-relative motion $Q \cdot Q$ ) term of $V$ to $M_{V}$ is

$$
\begin{aligned}
& M_{V}=\delta_{f, f^{\prime}} \frac{1}{16}(16 W+8 B-8 H-4 M)\left[\frac{\eta}{2 b^{2} \rho}\right]^{2}
\end{aligned}
$$

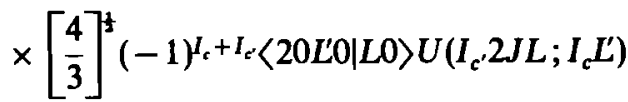

$$
\begin{aligned}
& \times G_{f}\left(\left(\lambda_{c} \mu_{c}\right) \kappa_{c} I_{c},\left(\lambda_{c^{\prime}} \mu_{c^{\prime}}\right) \kappa_{c^{\prime}} I_{c^{\prime}}\right)\left\langle\mathscr{R}_{L}^{(Q 0)}\left|\left(b_{1}+b_{2} \chi R^{2}\right) R^{2} \exp \left[-\chi R^{2}\right]\right| \mathscr{R}_{L^{\prime}}^{\left(Q^{\prime}\right)}\right\rangle,
\end{aligned}
$$

where

$$
\begin{aligned}
G_{f}\left(\left(\lambda_{c} \mu_{c}\right) \kappa_{c} I_{c},\left(\lambda_{c^{\prime}} \mu_{c^{\prime}}\right) \kappa_{c^{\prime}} I_{c^{\prime}}\right)=\sum_{\rho}\left\langle(11) 2 ;\left(\lambda_{c^{\prime}} \mu_{c^{\prime}}\right) \kappa_{c^{\prime}} I_{c^{\prime}} \|\left(\lambda_{c} \mu_{c}\right) \kappa_{c} I_{c}\right\rangle_{\rho} \\
\times\left\{(A-f) U\left((11)\left(\lambda_{f} \mu_{f}\right)\left(\lambda_{c} \mu_{c}\right)\left(\lambda_{A-f} \mu_{A-f}\right) ;\left(\lambda_{f} \mu_{f}\right)_{--} ;\left(\lambda_{c^{\prime}} \mu_{c^{\prime}}\right)_{-} \rho\right)\right. \\
\times(-1)^{1+\delta \mu_{f} .0}\left[C\left(\lambda_{f} \mu_{f}\right)\right]^{ \pm}+f U\left((11)\left(\lambda_{A-f} \mu_{A-f}\right)\left(\lambda_{c} \mu_{c}\right)\left(\lambda_{f} \mu_{f}\right) ;\right. \\
\left.\left.\left(\lambda_{A-f} \mu_{A-f}\right)_{--} ;\left(\lambda_{c^{\prime}} \mu_{c^{\prime}}\right)_{-} \rho\right)(-1)^{1+\delta \mu_{A-f^{\prime}} 0}\left[C\left(\lambda_{A-f} \mu_{A-f}\right)\right]^{\ddagger}\right\} .
\end{aligned}
$$

Finally, the third (internal-internal $Q \cdot Q$ ) term contributes

$$
\begin{aligned}
M_{V}= & \delta_{f, J^{\prime}} \delta_{I_{c} I_{e}} \delta_{L, L^{\prime}} \frac{1}{16}(16 W+8 B-8 H-4 M)\left[\frac{\eta}{2 b^{2} \rho}\right]^{\frac{1}{2}} \eta^{2} \\
& \times\left\langle\mathscr{R}_{L}^{(0)}\left|\left(\frac{1}{4}-\frac{1}{3} \chi R^{2}+\frac{1}{15} \chi^{2} R^{4}\right) \exp \left[-\chi R^{2}\right]\right| \mathscr{R}_{L}^{\left(Q^{\prime} 0\right)}\right\rangle
\end{aligned}
$$




$$
\begin{aligned}
& \times\left\{\delta_{\left(\lambda_{c} \mu_{c}\right) \kappa_{c}\left(\lambda_{c} \mu_{c}\right) \kappa_{c}} \frac{1}{2}\left[C\left(\lambda_{c} \mu_{c}\right)-C\left(\lambda_{f} \mu_{f}\right)-C\left(\lambda_{A-f} \mu_{A-f}\right)\right]\right. \\
& -\sum_{I_{f} I_{A-f}}\left\langle\left(\lambda_{f} \mu_{f}\right) I_{f} ;\left(\lambda_{A-f} \mu_{A-f}\right) I_{A-f} \|\left(\lambda_{c} \mu_{c}\right) \kappa_{c_{c}} \dot{I}_{c}\right\rangle \\
& \times\left\langle\left(\lambda_{f} \mu_{f}\right) I_{f} ;\left(\lambda_{A-f} \mu_{A-f}\right) I_{A-f} \|\left(\lambda_{c^{\prime}} \mu_{c^{\prime}}\right) \kappa_{c^{\prime}} I_{c^{\prime}}\right\rangle \\
& \left.\times \frac{1}{2}\left[I_{c}\left(I_{c}+1\right)-I_{f}\left(I_{f}+1\right)-I_{A-f}\left(I_{A-f}+1\right)\right]\right\} .
\end{aligned}
$$

\section{Results}

\subsection{THE MODEL PARAMETERS}

It is the aim of the present work to study the feasibility of a microscopic cluster model description of the ${ }^{12} \mathrm{C}+{ }^{12} \mathrm{C}$ resonances and to investigate the importance of the coupling of rearrangement channels to the ${ }^{12} \mathrm{C}+{ }^{12} \mathrm{C}$ channel for the rich complexity of the resonance fine structure. The emphasis is not on a detailed comparison of numerical calculations with experiment. Thus no search was made for optimum values of parameters to reproduce experimental data. Instead, only a minimum of adjustments have been made, and parameters have been chosen to be within a reasonable range of the values predicted from the underlying microscopic NN interaction model.

The threshold energies and excitation energies of the fragments are all taken from experiment. The harmonic oscillator size parameter $b$ is set to $1.7 \mathrm{fm}$. The NN interaction from which the folded potential has been constructed has been chosen to be the no. 2 potential of Hasegawa and Nagata ${ }^{20}$ ). (In this potential the radial forms of the even-state potentials have been fitted to the coordinate representation of the reaction matrices and the depth parameters have been determined so as to reproduce the binding energy of ${ }^{4} \mathrm{He}$. For the odd-state interaction they have used Tamagaki's potential which reproduces the $\alpha-\alpha$ scattering phase shifts reasonably well.) It is to be noted that our numerical results are sensitive only to the depth of the potential but are otherwise rather insensitive to the details of the effective interaction since only the coefficient $(16 W+8 B-8 H-4 M)$ appears in the matrix elements of our model.

Only two adjustments have been made in the final parameters of the folded potentials. Since the folded potentials often lead to a rotational spectrum which is too compressed, a multiplicative factor $c$ was introduced in the exponents of the gaussians $\exp \left(-c \gamma R^{2}\right)$, in order to strengthen slightly the fall off of the folded potentials. The values of $c$ chosen are $1.15,1.20$, and 1.15 for ${ }^{12} \mathrm{C}+{ }^{12} \mathrm{C}, \alpha+{ }^{20} \mathrm{Ne}$, and ${ }^{8} \mathrm{Be}+{ }^{16} \mathrm{O}$, respectively. This modification was introduced mainly to insure the proper characteristics for the ground-state rotational band, in particular the dominance of the $(\hat{\lambda} \mu)=(84)$ representation; cf. table 4 . In addition, in order to gain a consistent picture of the lowenergy spectrum of the $A=24$ system from the three different channels a small adjustment was made in the overall strength of the folded potential for each channel. The 
TABIE 4

Wave functions of the low-lying states

\begin{tabular}{|c|c|c|c|c|c|c|c|c|c|c|c|c|c|}
\hline$(i \mu) \kappa$ & $0_{1}^{+}$ & $0_{2}^{+}$ & $0_{3}^{+}$ & $2_{1}^{+}$ & $2_{2}^{+}$ & $2_{3}^{+}$ & $2_{4}^{+}$ & $4_{1}^{+}$ & $4_{2}^{+}$ & $4_{3}^{+}$ & $4_{4}^{+}$ & $4_{5}^{+}$ & $4_{6}^{+}$ \\
\hline$(84) 0$ & 0.75 & -0.27 & -0.41 & 0.80 & & 0.22 & & 0.68 & 0.49 & & 0.28 & & \\
\hline$(84) 2$ & & & & & 0.77 & & 0.38 & -0.38 & 0.64 & & -0.46 & & \\
\hline$(84) 4$ & & & & & & & & & & & & 0.65 & 0.52 \\
\hline (73)1 & & & & & & 0.66 & & -0.23 & & -0.50 & 0.34 & & \\
\hline (46)0 & -0.38 & -0.51 & & & 0.38 & & -0.49 & & -0.21 & & & -0.47 & 0.40 \\
\hline$(46) 2$ & & & & 0.24 & & & & & & & & 0.23 & 0.39 \\
\hline$(46) 4$ & & & & & & & & -0.36 & & 0.61 & 0.61 & & \\
\hline$(08) 0$ & 0.24 & 0.62 & 0.27 & & -0.26 & & 0.63 & & & & & 0.42 & -0.47 \\
\hline$(62) 0$ & -0.28 & 0.32 & -0.53 & -0.29 & & & & & & & & & \\
\hline$(62) 2$ & & & & & & & & & -0.23 & & & & \\
\hline (51)1 & & & & & & -0.48 & & & & 0.41 & -0.28 & & \\
\hline$(40) 0$ & & -0.25 & 0.61 & & & -0.26 & & & & & & & \\
\hline$(48) 0$ & & 0.24 & & & & & 0.23 & & & & & & \\
\hline
\end{tabular}

Only states with amplitudes greater than 0.2 are shown.

main component of the ground state of ${ }^{24} \mathrm{Mg}$ is the single (84) state of our basis. As pointed out earlier, the three (84) states represented in terms of a ${ }^{12} \mathrm{C}+{ }^{12} \mathrm{C}, \alpha+{ }^{20} \mathrm{Ne}$, and ${ }^{8} \mathrm{Be}+{ }^{16} \mathrm{O}$ cluster basis are thus identical states. A multiplicative factor $c^{\prime}$ was therefore introduced for the overall strength of the folded potential in each channel so that the position of a pure (84) $\mathrm{J}^{\pi}=0^{+}$state was identical when calculated with the three different cluster representations. With values of $c^{\prime}$ of $0.86735,1.0090$ and 0.89790 for ${ }^{12} \mathrm{C}+{ }^{12} \mathrm{C}, \alpha+{ }^{20} \mathrm{Ne}$, and ${ }^{8} \mathrm{Be}+{ }^{16} \mathrm{O}$, the initial expectation value of the pure (84) $0^{+}$ state was fixed at $6.0 \mathrm{MeV}$. This choice of the $(84) 0^{+}$matrix element is justified by the fact that the subsequent matrix diagonalization in the full basis of our model leads to a predicted ground-state energy very close to the experimental value.

\subsection{THE LOW-ENERGY SPECTRUM}

It is well known that the low-lying levels of ${ }^{24} \mathrm{Mg}$ can be classified into several rotational bands. The $0_{1}^{+}, 2_{1}^{+}$, and $4_{1}^{+}$states belong to the ground rotational band with $K^{\pi}=0^{+}$, the $2_{2}^{+}, 3_{1}^{+}$and $4_{2}^{+}$states to the $K^{\star}=2^{+}$band, and the $0_{2}^{+}$and $2_{4}^{+}$states to a $K^{\pi}=0^{+}$excited band.

The full three rearrangement channel calculation reproduces the observed spectrum reasonably well (fig. 1). However, the predicted spectrum is still too compressed, a property which is to be expected since our cluster model basis includes only states of $\left[4^{6}\right]$ space-symmetry of high $(\lambda \mu)$ with large intrinsic deformation and excludes mainly states with small $(\lambda \mu)$. If included, the latter, especially states of lower space symmetry, would tend to increase the rotational spacings. The leading components (with amplitudes $>0.2$ ) of the wave functions of the low-lying $0^{+}, 2^{+}, 4^{+}$states are shown in table 4. It is to be noted that, except for the (48) states, all the components shown in table 4 


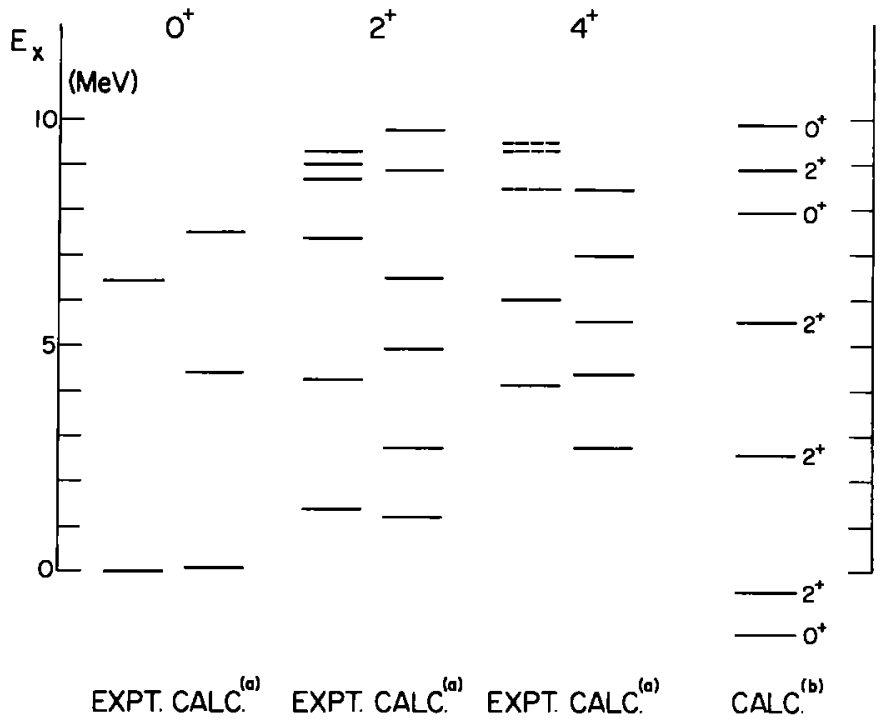

Fig. 1. Low-lying energy spectrum for ${ }^{24} \mathrm{Mg}$. (a) Calculations for the full ${ }^{12} \mathrm{C}+{ }^{12} \mathrm{C}, \alpha+{ }^{20} \mathrm{Ne}$, ${ }^{8} \mathrm{Be}+{ }^{16} \mathrm{O}$ cluster model basis with relative motion oscillator excitations, $12 \leqq Q \leqq 20$. (b) Calculations for a pure ${ }^{12} \mathrm{C}+{ }^{12} \mathrm{C}$ basis.

belong to the lowest shell-model configuration (cf. table 1). Our model space misses the additional (81) (54) (62) (35) (43) (51) (24) ${ }^{2}(32)(40)(13)$ and (02) states of the lowest shell-model configuration, but is wide enough that it encompasses the essential ingredients of the experimental data. Calculations with the use of a pure ${ }^{12} \mathrm{C}+{ }^{12} \mathrm{C}$ cluster basis only are also shown in fig. 1 (for states $0^{+}, 2^{+}$). It is to be noted that the agreement with experiment deteriorates with the use of the ${ }^{12} \mathrm{C}+{ }^{12} \mathrm{C}$ basis only. In particular, the pure ${ }^{12} \mathrm{C}+{ }^{12} \mathrm{C}$ basis does not reproduce the observed density of $2^{+}$states below 10 $\mathrm{MeV}$. The fact that the ground state is predicted to lie lower in the truncated pure ${ }^{12} \mathrm{C}+{ }^{12} \mathrm{C}$ basis is an artifice of the OCM approximation. States with $Q \geqq 14$, which lead to the depression of the $Q=12(\lambda \mu)=(84)$ ground state, belong predominantly to the ${ }^{12} \mathrm{C}+{ }^{12} \mathrm{C}$ cluster decomposition and are thus most effective in a pure ${ }^{12} \mathrm{C}+{ }^{12} \mathrm{C}$ basis. Their effectiveness is somewhat reduced in the full basis because their matrix connections to the $\alpha+{ }^{20} \mathrm{Ne}$ and ${ }^{8} \mathrm{Be}+{ }^{16} \mathrm{O}$ components of the (84) state are missing because of the assumed form of our effective interaction.

An extensive study of ${ }^{24} \mathrm{Mg}$ was carried out with an ${ }^{16} \mathrm{O}+2 \alpha \mathrm{OCM}$ cluster model by Kato and Bando ${ }^{12}$ ). In the lowest oscillator quanta this model space is identical with that of the $\alpha+{ }^{20} \mathrm{Ne}$ cluster basis and is generally more restricted than our mixed cluster model basis. The ${ }^{16} \mathrm{O}+2 \alpha$ model is thus incapable of predicting the $2_{3}^{+}$state at 7.35 $\mathrm{MeV}$, for example. We have also calculated the spectrum by restricting the basis to include the ${ }^{8} \mathrm{Be}+{ }^{16} \mathrm{O}$ channel only, in order to see whether the so-called "helicopter model" ${ }^{21}$ ) of ${ }^{24} \mathrm{Mg}$ may have some of the flavor of a microscopic basis. The results are 
far from encouraging. The helicopter model does not pay due attention to the Pauli principle. It includes many Pauli-forbidden components; and the potential parameters used differ widely from values which can be considered reasonable when inferred from a microscopic starting point.

\subsection{THE COULOMB BARRIER RESONANCE REGION}

To limit the dimensions of our basis, the harmonic oscillator excitations in the relative motion degrees of freedom have been restricted to $12 \leqq Q \leqq 20$. An expansion of the ${ }^{12} \mathrm{C}-{ }^{12} \mathrm{C}$ relative motion function in terms of oscillator functions with $Q=12$ 20 may be too restricted for good convergence. The possible molecular states, in particular, may require significant components with higher values of $Q$ in order to build up sufficient amplitudes in and beyond the Coulomb barrier-surface region of the ${ }^{12} \mathrm{C}+{ }^{12} \mathrm{C}$ system. The identification of possible candidates for the molecular resonances is therefore not immediately evident since our basis is not rich enough to give a detailed quantitative comparison between the theoretical model and experiment. The predicted excitation energies may be expected to be somewhat too high, and the predicted ${ }^{12} \mathrm{C}+{ }^{12} \mathrm{C}$ reduced width amplitudes must be expected to be too small in the surface region so that it is difficult to pinpoint their possible quasimolecular character. Nevertheless, it appears to be possible to identify a region in the predicted spectrum as the seat of the candidates for the molecular resonances with $\mathrm{J}^{x}=0^{+}, 2^{+}$and $4^{+}$.

Spectroscopic factors are used as a first rough indicator of the molecular character of our predicted states and will be used to single out the states of potential interest from the rich spectrum of predicted states. Our model predicts eight $0^{+}$, twenty-one $2^{+}$, and twenty-nine $4^{+}$states in the energy range from 16 to $27 \mathrm{MeV}$ of excitation energy in the $A=24$ system. To get a first qualitative idea as to which states may be candidates for the molecular resonances, we calculate the following quantity :

$$
\bar{S}=S_{0}\left(\sum_{i} S_{i}\right)
$$

Here, $S_{0}$ is the spectroscopic factor for the ${ }^{12} \mathrm{C}\left(0^{+}\right)+{ }^{12} \mathrm{C}\left(0^{+}\right)$channel and the $S_{i}$ are spectroscopic factors for some of the most important exit channels. For specific $J^{x}$, the sum over $i$ includes the specific channels defined below: For $0^{+}$states the sum over $i$ includes the $\alpha+{ }^{20} \mathrm{Ne}$ channels with (i) $I=0, L=0$; (ii) $I=2, L=2$; (iii) $I=4, L=4$; and the ${ }^{8} \mathrm{Be}+{ }^{16} \mathrm{O}$ channel with (iv) $I=0, L=0$.

For $2^{+}$states the sum over $i$ includes the ${ }^{12} \mathrm{C}+{ }^{12} \mathrm{C}$ channel with (i) $I_{1}=0, I_{2}=2, L$ $=0$; the $\alpha+{ }^{20} \mathrm{Ne}$ channels with (ii) $I=0, L=2$; (iii) $I=2, L=0$;(iv) $I=2, L=2$; (v) $I=2, L=4$; (vi) $I=4, L=2$; (vii) $I=4, L=4$; (viii) $I=4, L=6$; and the ${ }^{8} \mathrm{Be}+{ }^{16} \mathrm{O}$ channels with (ix) $I=0, L=2 ;$ (x) $I=2, L=0$.

For $4^{+}$states, the sum over $i$ includes the ${ }^{12} \mathrm{C}+{ }^{12} \mathrm{C}$ channel with (i) $I_{1}=0, I_{2}=2, L$ $=2$; the $\alpha+{ }^{20} \mathrm{Ne}$ channels with (ii) $I=0, L=4$; (iii) $I=2, L=2$; (iv) $I=2, L=4$; (v) 
$I=2, L=6$; (vi) $I=4, L=0$; (vii) $I=4, L=2$;(viii) $I=4, \mathrm{~L}=4$; (ix) $I=4, L=6$; (x) $I=4, L=8$; and the ${ }^{8} \mathrm{Be}+{ }^{16} \mathrm{O}$ channels with (xi) $I=0, L=4$; (xii) $I=2, L=2$. The spectroscopic factors are calculated as follows. The reduced width amplitude for the channel $i$ is defined by

$$
\begin{aligned}
& y_{i}(R)=\left\langle\mathscr{A} \frac{\delta\left(R_{f}-R\right)}{R^{2}}\left(\left(\phi_{J_{f}}^{\left(\lambda_{f} \mu_{f}\right)}(f) \times \phi_{I_{A-f}}^{\left.\lambda_{1-f} \mu_{A-f}\right)}(A-f)\right)_{I c} \times Y_{L}\left(\hat{R}_{f}\right)\right)_{J M} \mid \Psi_{J M}\right\rangle \\
& =\sum_{\beta} C_{\beta}\left\langle\mathscr { A } \frac { \delta ( R _ { f } - R ) } { R ^ { 2 } } \left(\left(\phi_{I_{f}}^{\left(\hat{\lambda}_{j} \mu_{f}\right)}(f) \times \phi_{\left.\left.I_{A-f}^{\left(\lambda_{A-f} \mu_{A-f}\right.}\right)(A-f)\right)_{I c}} \times Y_{L}\left(\hat{R}_{f}\right)\right)_{J M} \mid\right.\right. \\
& \left.\times \sqrt{\frac{1}{\beta}} \sum_{x^{\prime}} c_{\alpha^{\prime}} \psi_{a^{\prime}}\right\rangle
\end{aligned}
$$

Here the coefficients $C_{\beta}$ are the result of the full OCM matrix diagonalization and give the amplitudes of the basis states $(\sqrt{1 / \beta}) \sum_{\alpha} c_{\alpha} \psi_{\alpha}$, with norm eigenvalue $\beta$, in the expansion of $\Psi_{J M}$ [cf. eqs. (4) and (5)]. The spectroscopic amplitude, $A_{i \leftarrow \beta}$, for a particular eigenstate $\beta$ to channel $i\left(i=f ; I_{f} I_{A}-f_{c} L\right)$, is then defined by

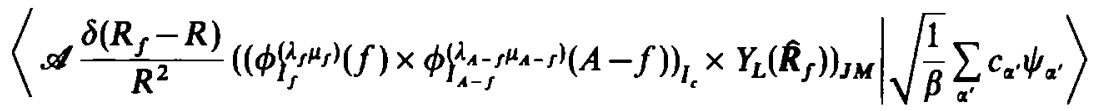

$$
\begin{aligned}
& =A_{i+\beta} \mathscr{R}_{L}^{(0)}(R) .
\end{aligned}
$$

The amplitude $A_{i-\beta}$ is given by

$$
A_{i \leftarrow \beta}=\sqrt{\beta} \sum_{\alpha} U_{i \alpha} c_{\alpha \cdot}
$$

Note that in $\alpha$ the fragment designation $f$ is fixed by the label $i$; the quantum numbers $(\lambda \mu)$ and $\kappa$ are fixed by their values in the parent state $\beta$. The sum over $\alpha$ in eq. (30) is therefore restricted to a sum over $\left(\lambda_{c} \mu_{c}\right)$ only. In eq. (29) the oscillator quantum number $Q$ is determined uniquely by the nature of the state $\beta$ and the fragment decomposition in the channel $i$. With these definitions the reduced width amplitude for the channel $i$, eq. (28), is given by

$$
y_{i}(R)=\sum_{\beta} C_{\beta} A_{i+\beta} \Re_{L}^{(Q 0)}(R)
$$

Note that the sum over $\beta$ drags along a sum over $Q$ since $Q$ and $\beta$ are in $1: 1$ correspondence. Note, also, that an extra factor of $\sqrt{ } 2$ is needed in $y_{i}(R)$ when $f$ and $A-f$ are identical ${ }^{12} \mathrm{C}$ fragments, and $I_{f} \neq I_{A-f}$.

The spectroscopic factors, $S_{i}$, are then given by

$$
S_{i}=\int \mathrm{d} R R^{2}\left|y_{i}(R)\right|^{2},
$$

and with these spectroscopic factors $\bar{S}$ of eq. (27) can be calculated. 


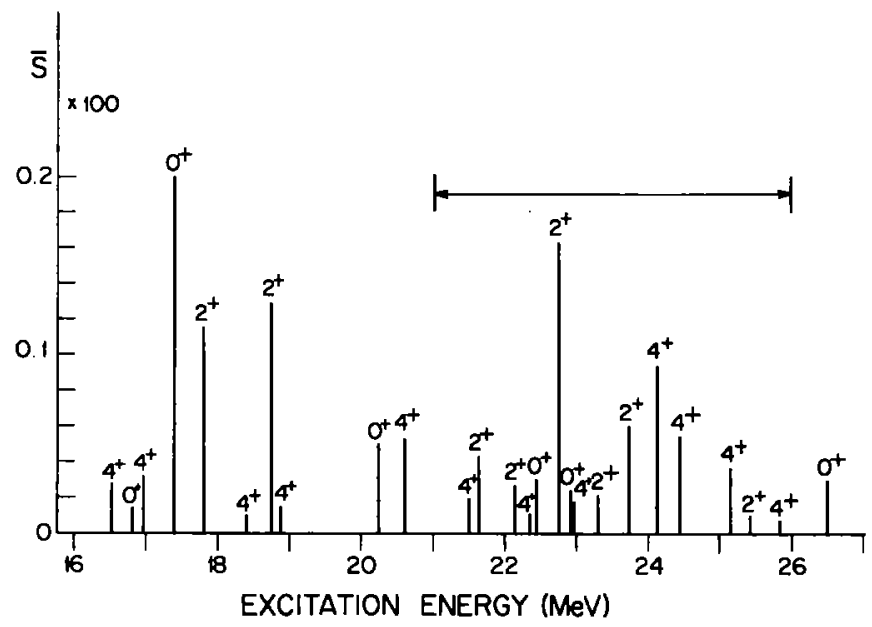

Fig. 2. Possible molecular states. The $S$-factors are defined in terms of spectroscopic factors by eq. (27) and are used as a qualitative indicator of possible molecular character. The region from $21-26 \mathrm{MeV}$ has been singled out as the potential seat of the sub-Coulomb resonances. Only states with significant $\boldsymbol{S}$-factors are shown. (In the $21-26 \mathrm{MeV}$ interval, e.g., the total number of $2^{+}$states is 10 .) For comparison with experiment, see fig. 2 of ref. ${ }^{26}$ ).

Fig. 2 shows the predicted $\mathrm{J}^{\pi}=0^{+}, 2^{+}, 4^{+}$states in the $16-27 \mathrm{MeV}$ excitation region, along with their predicted $\bar{S}$-values. Only states with significant values of $\bar{S}$ are indicated in the figure. Of the twenty-one $J^{x}=2^{+}$states predicted for this full energy range, e.g., only eight $2^{+}$states have predicted $\bar{S}$-factors large enough to show up in fig. 2. The experimentally observed $J^{\pi}=0^{+}, 2^{+}, 4^{+}$resonances lie in the $18-23 \mathrm{MeV}$ excitation $\left(E_{\text {c.m. }}=4-9 \mathrm{MeV}\right.$ ) region. Although it is not straightforward to identify possible candidates for the molecular resonances from among the states of fig. 2 , we focus on the states in the $21-26 \mathrm{MeV}$ region as the most likely candidates for the molecular resonances, for the following reasons:

(i) Most of these states have appreciable components with the larger oscillator excitations of our basis, specifically with $Q=14-18$. This is an appropriate characteristic of molecular states. (In an expanded basis including states with $Q>20$ these amplitudes would presumably shift to even higher $Q$-values and lead to relative-motion cluster functions with more peaking in the surface region.) As an illustration, some of the details of the structure of the two $0^{+}$states in the $21-26 \mathrm{MeV}$ region are shown in table 5. It is interesting to note that the representations $(14,6)$ and $(16,6)$ which have the largest amplitude in the $22.43 \mathrm{MeV}$ state occur only in the ${ }^{12} \mathrm{C}+{ }^{12} \mathrm{C}$ cluster basis. In addition, representations such as $(14,6),(16,6),(16,2)$, and $(18,2)$ can not be generated by quadrupole excitations acting on the ground state of ${ }^{24} \mathrm{Mg}$.

(ii) The predicted states below $21 \mathrm{MeV}$ consist predominantly of states of relatively pure $Q=14$. These are simple shell-model excitations and therefore not good molecular candidates. 
TABI.E 5

Wave functions of $0^{+}$molecular candidates

\begin{tabular}{ccccrr}
\hline$(\lambda \mu)$ & $0^{+}(22.43)$ & $0^{+}(22.93)$ & $(i \mu)$ & $0^{+}(22.43)$ & $0^{+}(22.93)$ \\
\hline$Q=14$ & & & $Q=18$ & & \\
$(10,4)_{1}$ & 0.14 & 0.25 & $(16,6)_{1}$ & 0.31 & -0.20 \\
$(10,4)_{2}$ & 0.13 & -0.18 & $(18,2)_{1}$ & 0.13 & 0.12 \\
$(10,4)_{3}$ & & -0.26 & $(18,2)_{2}$ & -0.18 & -0.11 \\
$(10,4)_{4}$ & & -0.27 & $(18,2)_{3}$ & 0.17 & 0.15 \\
$(12,0)$ & & -0.22 & $(14,4)_{1}$ & & 0.17 \\
$(82)_{1}$ & -0.21 & 0.13 & $(14,4)_{2}$ & & -0.15 \\
$(82)_{2}$ & 0.30 & 0.28 & $(16,0)$ & 0.12 & 0.17 \\
$(60)$ & 0.25 & & $(12,2)_{2}$ & \\
& & & $(10,0)$ & 0.10 & \\
$Q=16$ & & & & & \\
$(14,6)$ & 0.46 & & $(18,6)_{1}$ & 0.21 & 0.12 \\
$(16,2)_{1}$ & -0.17 & 0.27 & $(20,2)_{1}$ & & 0.11 \\
$(16,2)_{2}$ & -0.33 & 0.22 & $(20,2)_{2}$ & 0.15 & \\
$(12,4)_{1}$ & & 0.21 & $(16,4)_{1}$ & & \\
$(12,4)_{2}$ & & 0.21 & & & \\
$(12,4)_{4}$ & -0.10 & & & & \\
$(14,0)$ & & -0.20 & & & \\
$(10,2)_{2}$ & 0.13 & 0.21 & & & \\
\hline
\end{tabular}

Only states with amplitudes greater than 0.1 are shown. For the $22.43 \mathrm{MeV}$ state the percentages of $Q=12,14,16,18$ and 20 components are $0.59,26,41,23$ and 9.3. For the $22.93 \mathrm{MeV}$ state these percentages are $2.1,39,32,20$ and 7.0. Basis states $(\lambda \mu)_{1}$ with multiple occurences are ordered such that state $(\lambda \mu)_{1}$ has the largest overlap with the ${ }^{12} \mathrm{C}+{ }^{12} \mathrm{C}$ components, $(\lambda \mu)_{2}$ the next largest, etc. States $(\lambda \mu)_{i}$ which occur only in the ${ }^{12} \mathrm{C}+{ }^{12} \mathrm{C}$ channel are enumerated in decreasing order of $\beta$.

(iii) The excitation energies predicted in our truncated model basis must be expected to be somewhat too high since the inclusion in the basis of states with higher relative motion excitations $(Q>20)$ would lower the predicted energies, particularly of those states with significant components with $Q=18$ and 20 . The needed shift of about 3 $\mathrm{MeV}$ to lower energy seems reasonable.

(iv) The number of states predicted in the $5 \mathrm{MeV}$ interval from 21-26 MeV is in good agreement with the experimental resonances observed ${ }^{22-26}$ ) in the $5 \mathrm{MeV}$ interval from 18-23 MeV. Fig. 2 shows that there are two $0^{+}$, six $2^{+}$, and seven $4^{+}$states with significant $\bar{S}$-factors predicted for this $5 \mathrm{MeV}$ interval, in good agreement with the number of observed $0^{+}, 2^{+}$, and $4^{+}$peaks; [see fig. 2 of ref. ${ }^{26}$ ) and table 1 of ref. ${ }^{27}$ )].

We therefore identify these states as the candidates of the low- $J$ molecular resonance states. A definitive identification, however, should include a prediction of the widths of the resonances. 


\subsection{WIDTH ESTIMATES}

Because of our basis truncation the predicted relative-motion wave functions are least accurate in the asymptotic surface region, so that it is difficult to make reliable estimates of the widths. This difficulty is compounded by the differences between calculated and experimental energies.

An estimate of widths may be gained from the simple formula

$$
\Gamma_{i}=2 P_{i} \gamma_{\mathrm{w}}^{2} \theta_{i}^{2}(R),
$$

where $P_{l}$ is the Coulomb penetrability, $k R /\left[F_{L}^{2}(k R)+G_{L}^{2}(k R)\right], \gamma_{\mathrm{w}}^{2}=3 \hbar^{2} / 2 \mu R^{2}$ is the Wigner-limit value, and $\theta_{t}^{2}(R)$ is the dimensionless reduced width which is given in terms of the reduced width amplitude, $y_{t}(R)$, by

$$
\theta_{l}(R)=\left[\frac{1}{3} R^{3}\right]^{\frac{1}{2}} y_{i}(R)
$$

If our predictions were to give a reasonably accurate functional form for $y_{i}(R)$ for a wide enough interval of the interior region, the value of $\theta_{l}(R)$ at the channel radius could be obtained by a suitable correction of the tail behavior of $\left.y_{i}(R)\left[\mathrm{ref}{ }^{28}\right)\right]$. Since our model space is too restricted to give a reliable value of $y_{i}(R)$ near the surface region, it is not possible to apply the above prescription. Instead, we make the simple assumption that $\theta_{i}^{2}(R)$ is given through the spectroscopic factor by $\theta_{l}^{2}(R)=\frac{2}{3} S_{i}\left[\right.$ ref. $\left.\left.{ }^{28}\right)\right]$. This is apt to overestimate the reduced width. The penetrabilities have been calculated for the various decay channels. In the ${ }^{12} \mathrm{C}+{ }^{12} \mathrm{C}$ Coulomb-barrier region the penetrabilities for the various ${ }^{12} \mathrm{C}+{ }^{12} \mathrm{C}$ and ${ }^{8} \mathrm{Be}+{ }^{16} \mathrm{O}$ channels are extremely sensitive functions of the excitation energy. The penetrabilities which have been used are therefore those for the experimentally observed excitation energies. (This implies that our association of predicted resonance fine structure components with the observed ones matches the appropriate partners.) For the $\alpha+{ }^{20} \mathrm{Ne}$ channels the energy dependence of the penetrabilities is less pronounced. The partial width estimates of the $\alpha+{ }^{20} \mathrm{Ne}$ channels are thus less sensitive to the correct assignments of fine structure components.

Table 6 lists the estimated partial widths for a few of the predicted resonances (cf. fig. 2 ) by matching them with observed resonances through rather arbitrary assignments. (Note, e.g., that we have matched three of the prominent higher predicted $4^{+}$resonances with three of the observed resonances in the corresponding upper part of the observed low- $J$ resonance region). Table 6 shows that the ${ }^{12} \mathrm{C}$ and ${ }^{8} \mathrm{Be}$ partial widths are seemingly too small. The smallness of the ${ }^{12} \mathrm{C}\left(0^{+}\right)+{ }^{12} \mathrm{C}\left(0^{+}\right)$partial widths, in particular, casts some question as to a ${ }^{12} \mathrm{C}+{ }^{12} \mathrm{C}$ molecular interpretation of these resonances. The fact that our predicted wave functions do not have sufficient ${ }^{12} \mathrm{C}+{ }^{12} \mathrm{C}$ amplitude may, however, be related to the fact that the model space which was used is too restricted to give a full description of the ${ }^{12} \mathrm{C}+{ }^{12} \mathrm{C}$ resonances. The partial widths for the $\alpha+{ }^{20} \mathrm{Ne}$ channels are of the right order of magnitude to yield the observed total 
TABI E 6

Estimated widths (in keV)

\begin{tabular}{lclllrrrr}
\hline $\begin{array}{c}E_{\mathrm{z}} \text { (calc) } \\
(\mathrm{MeV})\end{array}$ & $\begin{array}{c}E_{\text {c.m. }}(\mathrm{exp}) \\
\left.(\mathrm{MeV})^{\mathrm{a.b}}\right)\end{array}$ & $J^{*}$ & $\begin{array}{c}\Gamma \text { (exp) } \\
\left.(\mathrm{keV})^{\mathrm{b}}\right)\end{array}$ & $\Gamma\left(\mathrm{C}_{0}\right)$ & $\Gamma\left(\alpha_{0}\right)$ & $\Gamma\left(\alpha_{1}\right)$ & $\Gamma\left(\alpha_{2}\right)$ & $\Gamma\left(\mathrm{Be}_{0}\right)$ \\
\hline 22.43 & 4.25 & $0^{+}$ & & 0.02 & 2 & 15 & 1 & 0.006 \\
22.93 & 5.80 & $0^{+}$ & & 0.7 & 7 & 8 & 8 & 1 \\
22.75 & 5.64 & $2^{+}$ & & 0.4 & 18 & 88 & 98 & 0.1 \\
23.73 & 6.64 & $2^{+}$ & 100 & 1 & 1 & 32 & 96 & 1 \\
24.13 & 6.85 & $4^{+}$ & 125 & 1 & 58 & 86 & 69 & 0.2 \\
24.45 & 7.71 & $4^{+}$ & 125 & 0.3 & 78 & 430 & 440 & 0.05 \\
25.15 & 7.85 & $4^{+}$ & & 0.5 & 66 & 80 & 200 & 0.1 \\
\hline
\end{tabular}

-) Ref. $\left.{ }^{27}\right)$. b) Ref. ${ }^{23}$ ).

$\Gamma\left(x_{1}\right)$ and $\Gamma\left(\alpha_{2}\right)$ stand for the estimated widths of the decays to the $2^{+}$and $4^{+}$(1st and 2 nd excited) states of ${ }^{20} \mathrm{Ne}$.

widths. Some of the overestimates may be related to our prescription for the reduced width amplitude which is apt to lead to such overestimates. Although the nucleon channels have not been explicitly included in our coupled channel basis, the overlaps between the ${ }^{12} \mathrm{C}+{ }^{12} \mathrm{C}$ components of our basis and the $\mathrm{p}+{ }^{23} \mathrm{Na}$ cluster functions ${ }^{10}$ ) permit us to make a rough order of magnitude estimate and indicate that the partial widths to the proton channel and some of the low-lying states in ${ }^{23} \mathrm{Na}$ can be of the same order of magnitude as the partial widths for the $\alpha+{ }^{20} \mathrm{Ne}$ channels.

\section{Conclusions}

Although a rich basis, including rearrangement channels based on $\alpha+{ }^{20} \mathrm{Ne}$ and ${ }^{8} \mathrm{Be}+{ }^{16} \mathrm{O}$ fragment decompositions, has been used to study the low- $J$ Coulombbarrier resonance region in the ${ }^{12} \mathrm{C}+{ }^{12} \mathrm{C}$ excitation functions, the basis used in the present investigation may not be rich enough in the oscillator excitations of the relative motion degrees of freedom to give a detailed quantitative description of these resonances. Nevertheless, it appears to be possible to identify a $5 \mathrm{MeV}$ region in the predicted spectrum as the potential seat of the $\mathrm{J}^{\pi}=0^{+}, 2^{+}, 4^{+}$resonances observed in the excitation functions of the ${ }^{12} \mathrm{C}+{ }^{12} \mathrm{C}$ resonances. The number of $0^{+}, 2^{+}$, and $4^{+}$ states and the approximate spacing of these resonances are in qualitative agreement with the experimental findings. For this purpose it appears to be important to couple ${ }^{12} \mathrm{C}+{ }^{12} \mathrm{C}, \alpha+{ }^{20} \mathrm{Ne}$, and ${ }^{8} \mathrm{Be}+{ }^{16} \mathrm{O}$ channels. A model built on a pure ${ }^{12} \mathrm{C}+{ }^{12} \mathrm{C}$ cluster basis does not appear to be rich enough to yield the full complexity of the observed resonance fine structure. The estimates of the total widths are of the right order of magnitude. Estimates of the ${ }^{12} \mathrm{C}\left(0^{+}\right)+{ }^{12} \mathrm{C}\left(0^{+}\right)$partial widths, however, indicate that our calculated model wave functions do not predict enough ${ }^{12} \mathrm{C}+{ }^{12} \mathrm{C}$ reduced width 
amplitude in the surface region, where these amplitudes are not sufficiently well developed to be classified as molecular in character. The basis of the present investigation must be expanded to include higher oscillator excitations in the relative motion degrees of freedom. However, these initial results make it doubtful whether such an expansion of the basis will lead to quasibound states of true molecular character. Although the present study leads us to conclude that a microscopic cluster model can account for the major features of the observed resonances in the Coulomb barrier region of the ${ }^{12} \mathrm{C}+{ }^{12} \mathrm{C}$ system, it may be more accurate to characterize the quasibound states responsible for these resonances as quasimolecular rather than true molecular states.

One of the authors (Y.S.) acknowledges the Nishina Memorial Foundation for financial support and the University of Michigan for its hospitality. We thank J. P. Draayer for valuable aid with the computational effort, $W$. Zahn for making available his norm and overlap codes and for his continued interest in this problem, and Y. Kondo for his code of Coulomb wave functions.

\section{References}

1) D. A. Bromley, J. A. Kuehner and E. Almqvist, Phys. Rev. Lett. 4 (1960) 365, 515

2) D. A. Bromley, in Nuclear molecular phenomena ed. N. Cindro (North-Holland, Amsterdam, 1978)

3) R. H. Siemssen, Proc. INS-IPCR Symp. on cluster structure of nuclei and transfer reactionsinduced by heavy-ions, ed. H. Kamitsubo, I. Kohno and T. Marumori (Tokyo, 1975) p. 233

4) B. Imanishi, Nucl. Phys. A125 (1969) 33

5) J. Y. Park, W. Scheid and W. Greiner, Phys. Rev. C10 (1974) 967;C16 (1977) 2276

6) Y. Kondo, T. Matsuse and Y. Abe, Prog. Theor. Phys. 59 (1978) 465; Phys. Rev. C19 (1979) 1356, 1365; Prog. Theor. Phys. Suppl. 68 (1980) 303; also Y. Abe, in Nuclear molecular phenomena ed. N. Cindro (North-Holland, Amsterdam, 1978)

7) H. Feshbach, J. de Phys. Collog. 37 (1976) C5-177;

O. Tanimura and T. Tazawa, Phys. Reports 61 (1980) 253;

R. Koennecke, W. Greiner, J. Y. Park and W. Scheid, Proc. Int. Conf. on the resonant behavior of heavy-ion systems, ed. G. Vourvopoulos (Aegean Sea, Greece, 1980) National Printing Office, Athens, 1981

8) A. Tohsaki-Suzuki, Prog. Theor. Phys. 60 (1978) 1013

9) S. Saito, Prog. Theor. Phys. 41 (1969) 705

10) K. T. Hecht, E. J. Reske, T. H. Seligman and W. Zahn, Nucl. Phys. A356 (1981) 146

11) K. T. Hecht, H. M. Hofmann and W. Zahn, Phys. Lett. 103B (1981) 92

12) K. Kato and H. Bando, Prog. Theor. Phys. 62 (1979) 644

13) Y. Fujiwara, H. Horiuchi, K. Ikeda, M. Kamimura, K. Kato, Y. Suzuki and E. Uegaki, Prog. Theor. Phys. Suppl. 68 (1980) 29

14) M. Ohkubo, K. Kato and H. Tanaka, Prog. Theor. Phys. 67 (1982) 207

15) Y. Suzuki, Prog. Theor. Phys. 55 (1976) 1751 ; 56 (1976) 111

16) Y. Suzuki, T. Ando and B. Imanishi, Nucl. Phys. A295 (1978) 365;

Y. Suzuki and B. Imanishi, Phys. Rev. C23 (1981) 2414

17) Y. Fujiwara, H. Horiuchi and R. Tamagaki, Prog. Theor. Phys. 61 (1979) 1629;

Y. Fujiwara, Prog. Theor. Phys. 62 (1979) 122, 138

18) Y. Suzuki, E. J. Reske and K. T. Hecht, Nucl. Phys. A381 (1982) 77

19) Y. Goto and H. Horiuchi, Prog. Theor. Phys. 62 (1979) 662

20) A. Hasegawa and S. Nagata, Prog. Theor. Phys. 45 (1971) 1786

21) A. A. Pilt and C. Wheatley, Phys. Lett. 76B (1978) 11 
22) W. Galster, W. Treu, P. Dück, H. Frőhlich and H. Voit, Phys. Rev. C15 (1977) 950

23) K. A. Erb, R. R. Betts, S. K. Korotky, M. M. Hindi, P. P. Tung, M. W. Sachs, S. J. Willett and D. A. Bromley, Phys. Rev. C22 (1980) 507

24) R. Wada, T. Murakami, E. Takada, M. Fukuda and K. Takimoto, Phys. Rev. C22 (1980) 557

25) K. U. Kettner, H. Lorenz-Wirzba and C. Rolfs, Z. Phys. A298 (1980) 65

26) K. A. Erb, D. A. Bromley and J. Weneser, Comm. Nucl. Part. Phys. A8 (1978) 111

27) K. A. Erb and D. A. Bromley, Phys. Rev. C23 (1981) 2781

28) H. Horiuchi and Y. Suzuki, Prog. Theor. Phys. 49 (1973) 1974 